\title{
A note on a transform to self-inverse sequences
}

\author{
Altan Erdoğan (10 \\ Gebze Technical University, Gebze, Kocaeli, Turkey
}

\begin{abstract}
The sequences which are fixed by the binomial transform are called self-inverse sequences. In this paper, an identity satisfied by Fibonacci numbers is modified to provide a transform which maps a specific subset of sequences to self-inverse sequences bijectively. The image of some classes of sequences under this transform are explicitly found which provides a new formulation and a class of examples of self-inverse sequences. A criterion for the solutions of some difference equations to be self-inverse is also given.
\end{abstract}

Mathematics Subject Classification (2020). 05A10, 05A19, 11B65, 11B37

Keywords. binomial transform, self-inverse sequences, difference equations

\section{Introduction}

Let $\mathcal{A}$ denote the set of sequences $\mathbf{d}=\left\{d_{n}\right\}_{n \geq 0}$ over a field of characteristic zero. The binomial transform, $\mathcal{B}$ of a sequence $\mathbf{d} \in \mathcal{A}$ is defined as

$$
\mathcal{B}: \mathcal{A} \rightarrow \mathcal{A}, \mathcal{B}(\mathbf{d})_{n}=\sum_{k=0}^{n}\left(\begin{array}{l}
n \\
k
\end{array}\right)(-1)^{k} d_{k}, n \geq 0 .
$$

The ordinary generating function of a sequence $\mathbf{d}=\left\{d_{n}\right\}_{n \geq 0}$ is the formal power series $f(T)=\sum_{n=0}^{\infty} d_{n} T^{n}$. In this case we simple write

$$
\mathbf{d} \sim f(T) .
$$

The generating function of a sequence $\mathbf{d}$ and of its binomial transform are related to each other; for any sequence $\mathbf{d}$,

$$
\mathbf{d} \sim f(T) \Longrightarrow \mathcal{B}(\mathbf{d}) \sim \frac{1}{1-T} f\left(\frac{-T}{1-T}\right)
$$

(See [3] or [4]). It is easy to see that $\mathcal{B}^{2}(\mathbf{d})=\mathbf{d}$, so that the eigenvalues of $\mathcal{B}$ are \pm 1 . The eigenspaces of $\mathcal{B}$, i.e. sequences for which $\mathcal{B}(\mathbf{d})= \pm \mathbf{d}$ are of common interest. A sequence $\mathbf{d} \in \mathcal{A}$ is said to be self-inverse (or invariant under $\mathcal{B}$ ) if $\mathcal{B}(\mathbf{d})=\mathbf{d}$. We denote the subset of self-inverse sequences by $\mathcal{A}^{+}$. Note that $\mathcal{A}^{+}$is a subspace of $\mathcal{A}$. Typical examples of self-inverse sequences are

$$
\left\{1 / 2^{n}\right\}_{n \geq 0},\left\{n F_{n-1}\right\}_{n \geq 0},\left\{(-1)^{n} B_{n}\right\}_{n \geq 0},
$$

where $F_{n}$ and $B_{n}$ denote the $n$-th Fibonacci and Bernoulli numbers respectively with $F_{0}=0$. An extensive list of examples of both eigenspaces of $\mathcal{B}$ with related recurrence

Email address: alerdogan@gtu.edu.tr

Received: 25.07.2020; Accepted: 04.03.2021 
relations are proved in [4]. Also some congruences modulo prime powers involving both eigenspaces are given in [5]. Another interesting result is that $\mathcal{A}$ is equal to the direct sum of eigenspaces of $\mathcal{B}$ which can be proved by using the matrix representation of $\mathcal{B}$, [6]. In this paper, we shall restrict to eigenspace of $\mathcal{B}$ corresponding to the eigenvalue 1 .

There is another convention for the binomial transform where the term $(-1)^{k}$ in the summation in $\mathcal{B}$ is omitted, but we will adopt the above convention. The reader is referred to [1] for the alternative convention and also a general exposition on transforms of integer sequences.

Let $\mathcal{A}_{0}$ denote the subspace of $\mathcal{A}$ consisting of sequences $\mathbf{d}=\left\{d_{n}\right\}_{n \geq 0}$ for which $d_{0}=0$. We denote the subspace of self-inverse sequences in $\mathcal{A}_{0}$ by $\mathcal{A}_{0}^{+}$. Note that for any $\mathbf{d} \in \mathcal{A}_{0}^{+}$, we necessarily have $d_{0}=d_{1}=0$. Let $\mathcal{H}$ be the transform on $\mathcal{A}_{0}$ defined as

$$
\begin{aligned}
\mathcal{H}: \mathcal{A}_{0} & \rightarrow \mathcal{A}_{0}, \\
\mathcal{H}(\mathbf{c})_{n} & =\sum_{k=0}^{\lfloor n / 2\rfloor}\left(\begin{array}{c}
n-k-1 \\
k-1
\end{array}\right)(-1)^{k} c_{k}, n \geq 2,
\end{aligned}
$$

where $\lfloor x\rfloor$ denotes the greatest integer less than or equal to $x$. Note that the binomial term corresponding to $k=0$ is $\left(\begin{array}{c}n-1 \\ -1\end{array}\right)$ which may be defined in different ways. But we don't need to care about this ambiguity since we apply $\mathcal{H}$ on sequences for which $c_{0}=0$. So we actually have that

$$
\mathcal{H}(\mathbf{c})_{0}=\mathcal{H}(\mathbf{c})_{1}=0, \mathcal{H}(\mathbf{c})_{n}=\sum_{k=1}^{\lfloor n / 2\rfloor}\left(\begin{array}{c}
n-k-1 \\
k-1
\end{array}\right)(-1)^{k} c_{k}, n \geq 2, \mathbf{c} \in \mathcal{A}_{0} .
$$

The main result of this paper is the following theorem.

Theorem 1.1. For any $\mathbf{c} \in \mathcal{A}_{0}$, the sequence $\left\{n \mathcal{H}(\mathbf{c})_{n}\right\}_{n \geq 0}$ is a self-inverse sequence. Moreover the map

is a bijection.

$$
\mathcal{A}_{0} \rightarrow \mathcal{A}_{0}^{+}, \mathbf{c} \mapsto\left\{n \mathcal{H}(\mathbf{c})_{n}\right\}_{n \geq 0}
$$

This result is a characterization of self-inverse sequences in $\mathcal{A}_{0}^{+}$. For example the sequence $\mathbf{c}$ for which $c_{0}=0, c_{k}=(-1)^{k}$ for $k \geq 1$ maps to $\left\{n F_{n-1}\right\}_{n \geq 0}$ where $\left\{F_{n}\right\}$ is the Fibonacci sequence (We take $F_{-1}=F_{0}=0$ ). It is possible to recover $\mathcal{A}^{+}$from $\mathcal{A}_{0}^{+}$since we have the following;

$$
\left(0,0, d_{2}, d_{3}, \ldots\right) \in \mathcal{A}_{0}^{+} \quad \text { if and only if }\left(d_{2}, d_{3}-d_{2}, d_{4}-d_{3}, \ldots\right) \in \mathcal{A}^{+},
$$

This follows by [4, Corollary 3.1-3.2].

The organization of the paper is as follows. In Section 2 we study the ordinary generating functions and as a result prove above theorem. The idea of the proof is expressing the difference operator as a conjugation by a differential operator applied on ordinary generating functions.

Section 3 is devoted to characterization of specific types of self-inverse sequences. We shall focus on two types of sequences. We find a basis for self-inverse sequences of polynomial type in $\mathcal{A}_{0}^{+}$. We also study sequences which satisfy specific recurrence relations. 


\section{Main theorem}

We define the formal differential operator $D$ on the ring of formal power series as $D=T \frac{d}{d T}$, explicitly if $f(T)=\sum_{n=0}^{\infty} d_{n} T^{n}$ then

$$
D f(T)=\sum_{n=1}^{\infty} n d_{n} T^{n}
$$

By abuse of notation we may also use $D$ to denote the corresponding transform on $\mathcal{A}$ and write $D(\mathbf{d})=\left\{n d_{n}\right\}_{n \geq 0}$ for any $\mathbf{d} \in \mathcal{A}$. Clearly $D$ does not have an inverse. But if we restrict $D$ to $\mathcal{A}_{0}$, then $D$ is invertible with inverse $D^{-1}$ where

$$
\begin{aligned}
& D^{-1}: \mathcal{A}_{0} \rightarrow \mathcal{A}_{0}, \\
& D^{-1}(\mathbf{d})_{0}=0, D^{-1}(\mathbf{d})_{n}=d_{n} / n \text { for } n \geq 1
\end{aligned}
$$

In terms of generating functions we have

$$
f(T)=\sum_{n=1}^{\infty} d_{n} T^{n} \Longrightarrow D^{-1} f(T)=\sum_{n=1}^{\infty} \frac{d_{n}}{n} T^{n}
$$

We define another transform $\mathcal{B}^{\prime}$ on $\mathcal{A}_{0}$ as $\mathcal{B}^{\prime}=D^{-1} \mathcal{B} D$, explicitly for any $\mathbf{d} \in \mathcal{A}_{0}$

$$
\mathcal{B}^{\prime}(\mathbf{d})_{0}=0, \mathcal{B}^{\prime}(\mathbf{d})_{n}=\sum_{k=1}^{n}\left(\begin{array}{l}
n-1 \\
k-1
\end{array}\right) d_{k}(-1)^{k} \text { for } n \geq 1 .
$$

Equivalently, we can define $\mathcal{B}^{\prime}$ by $\mathcal{B}^{\prime}(\mathbf{d})_{n}=\mathcal{B}(\mathbf{d})_{n}-\mathcal{B}(\mathbf{d})_{n-1}$ for $n \geq 1$. This difference operator in relation to self-inverse sequences allows one to obtain formulas involving harmonic numbers and Stirling numbers, [2].

But we emphasize the relation $\mathcal{B}^{\prime}=D^{-1} \mathcal{B} D$ on $\mathcal{A}_{0}$ which simply implies that

$$
\mathcal{B}^{\prime}(\mathbf{d})=\mathbf{d} \text { if and only if } \mathcal{B}(D(\mathbf{d}))=D(\mathbf{d})
$$

for $\mathbf{d} \in \mathcal{A}_{0}$. We say that $\mathbf{d}$ is invariant under $\mathcal{B}^{\prime}$ if $\mathcal{B}^{\prime}(\mathbf{d})=\mathbf{d}$. So we shall study the invariant sequences under $\mathcal{B}^{\prime}$ and then relate to $\mathcal{B}$. Note that if $\mathbf{d} \in \mathcal{A}_{0}$ is invariant under $\mathcal{B}\left(\right.$ or $\mathcal{B}^{\prime}$ ) then necessarily $d_{0}=d_{1}=0$. We have a simple description of $\mathcal{B}^{\prime}$ in terms of the ordinary generating functions.

Lemma 2.1. Let $\mathbf{d} \in \mathcal{A}_{0}$ and $\mathbf{d} \sim f(T)$. Then

$$
\mathcal{B}^{\prime}(\mathbf{d}) \sim f\left(\frac{-T}{1-T}\right),
$$

where $\frac{1}{1-T}=\sum_{n=0}^{\infty} T^{n}$.

Proof. Recall that for any $\mathbf{a} \in \mathcal{A}$, if $\mathbf{a} \sim f(T)$ then

$$
\mathcal{B}(\mathbf{a}) \sim \frac{1}{1-T} f\left(\frac{-T}{1-T}\right) .
$$

Since $D(\mathbf{d}) \sim T f^{\prime}(T)$ we have that

$$
\mathcal{B} D(\mathbf{d}) \sim \frac{-T}{(1-T)^{2}} f^{\prime}\left(\frac{-T}{1-T}\right) .
$$

But by chain rule we also see that

$$
D\left[f\left(\frac{-T}{1-T}\right)\right]=\frac{-T}{(1-T)^{2}} f^{\prime}\left(\frac{-T}{1-T}\right) .
$$

Applying $D^{-1}$ we obtain

$$
\mathcal{B}^{\prime}(\mathbf{d})=D^{-1} \mathcal{B} D(\mathbf{d}) \sim f\left(\frac{-T}{1-T}\right) .
$$


In particular, for any sequence $\mathbf{d} \sim f(T)$ we have that

$$
\mathcal{B}^{\prime}(\mathbf{d})=(\mathbf{d}) \Longleftrightarrow f(T)=f\left(\frac{-T}{1-T}\right) .
$$

So we shall investigate the formal power series which are symmetric in $T$ and $-T /(1-T)$. The simplest nonzero power series symmetric in $T$ and $-T /(1-T)$ is

$$
T+\frac{-T}{1-T}=T \frac{-T}{1-T}=\frac{-T^{2}}{1-T} \text {. }
$$

As a consequence any sequence in $\mathcal{A}_{0}$ with an ordinary generating function of the form $f\left(-T^{2} /(1-T)\right)$ for some $f(T)$ is invariant under $\mathcal{B}^{\prime}$.

Example 2.2. Let $\mathbf{d} \sim f(T)=\ln ^{2}(1-T) / 2$ where $\ln (1-T)=-\sum_{n=1}^{\infty} \frac{T^{n}}{n}$, so that $\mathbf{d} \in \mathcal{A}_{0}$. We may easily verify that

$$
f(T)=f(-T /(1-T)),
$$

which implies that $\mathcal{B}^{\prime}(\mathbf{d})=\mathbf{d}$. But by computing the square of $\ln (1-T)$ we can also see that

$$
f(T)=\sum_{k=2}^{\infty} \frac{H_{k-1}}{k} T^{k}
$$

where $H_{k-1}=1+1 / 2+1 / 3+\ldots+1 /(k-1)$ are the harmonic numbers. Then we have that $D(\mathbf{d})=\left\{0,0, H_{1}, H_{2}, \ldots\right\}$. By $(2.1), D(\mathbf{d})$ is invariant under $\mathcal{B}$, i.e. we have the recurrence

$$
\sum_{k=2}^{n}\left(\begin{array}{l}
n \\
k
\end{array}\right)(-1)^{k} H_{k-1}=H_{n-1}, n \geq 2 .
$$

Note that this fact can also be derived by applying (1.2) to the self-inverse sequence $\{1,1 / 2,1 / 3, \ldots\}$.

Theorem 2.3. For any $\mathbf{c} \in \mathcal{A}_{0}$, the sequence $\left\{n \mathcal{H}(\mathbf{c})_{n}\right\}_{n \geq 0}$ is a self-inverse sequence. Moreover the map

is a bijection.

$$
\mathcal{A}_{0} \rightarrow \mathcal{A}_{0}^{+}, \mathbf{c} \mapsto\left\{n \mathcal{H}(\mathbf{c})_{n}\right\}_{n \geq 0}
$$

Proof. Let $\mathbf{c} \in \mathcal{A}_{0}$ and $\mathbf{c} \sim g(T)$. For the first part of the theorem by (2.1) it is enough to prove that $\mathcal{H}(\mathbf{c})$ is invariant under $\mathcal{B}^{\prime}$. Let $\mathbf{d}$ be the sequence for which $\mathbf{d} \sim g\left(-T^{2} /(1-T)\right)$. By Lemma 2.1 and the conclusion following it, $\mathbf{d}$ is invariant under $\mathcal{B}^{\prime}$. Now we will show that indeed $\mathbf{d}=\mathcal{H}(\mathbf{c})$. Let $g(T)=\sum_{k=1}^{\infty} c_{k} T^{k}$. Then

$$
\begin{aligned}
g\left(\frac{-T^{2}}{1-T}\right) & =\sum_{k=1}^{\infty} c_{k}\left(\frac{-T^{2}}{1-T}\right)^{k}=\sum_{k=1}^{\infty} c_{k}(-1)^{k} T^{2 k} \frac{1}{(1-T)^{k}} \\
& =\sum_{k=1}^{\infty} c_{k}(-1)^{k} \sum_{l=k}^{\infty}\left(\begin{array}{l}
l-1 \\
k-1
\end{array}\right) T^{l+k} .
\end{aligned}
$$

In the last equality we use that $1 /(1-T)^{k}=\sum_{l=k}^{\infty}\left(\begin{array}{l}l-1 \\ k-1\end{array}\right) T^{l-k}$ for $k \geq 1$ which can be proven inductively. We set $n=l+k$ and obtain that

$$
g\left(\frac{-T^{2}}{1-T}\right)=\sum_{k=1}^{\infty} c_{k}(-1)^{k} \sum_{n=2 k}^{\infty}\left(\begin{array}{c}
n-k-1 \\
k-1
\end{array}\right) T^{n} .
$$

We interchange the indices $k$ and $n$ and deduce that

$$
g\left(\frac{-T^{2}}{1-T}\right)=\sum_{n=2}^{\infty}\left(\sum_{k=1}^{\lfloor n / 2\rfloor}\left(\begin{array}{c}
n-k-1 \\
k-1
\end{array}\right) c_{k}(-1)^{k}\right) T^{n},
$$


which completes the proof of the first part of the theorem.

For the second part, first we note that the map $\mathbf{c} \mapsto\left\{n \mathcal{H}(c)_{n}\right\}_{n \geq 0}$ on $\mathcal{A}_{0}$ is linear. If $\mathcal{H}(\mathbf{c})(n)=0$ for all $n \geq 2$ then by induction it follows that $c_{k}=0$ for $k \geq 1$. So the map $\mathbf{c} \mapsto\left\{n \mathcal{H}(\mathbf{c})_{n}\right\}_{n \geq 0}$ on $\mathcal{A}_{0}$ is injective.

Now we prove the surjectivity. Again by (2.1) it is enough to show that for any sequence $\mathbf{d} \in \mathcal{A}_{0}$ which is invariant under $\mathcal{B}^{\prime}$ there exists $\mathbf{c} \in \mathcal{A}_{0}$ such that $\mathcal{H}(\mathbf{c})=\mathbf{d}$. Let $\mathbf{d}$ be invariant under $\mathcal{B}^{\prime}$. Note that we necessarily have $d_{0}=d_{1}=0$. Consider the subsequence $\left\{d_{2 m}\right\}_{m \geq 1}$. We can uniquely determine $\left\{c_{k}\right\}_{k \geq 1}$ such that

$$
d_{2 m}=\sum_{k=1}^{m}\left(\begin{array}{c}
2 m-k-1 \\
k-1
\end{array}\right) c_{k}(-1)^{k}
$$

recursively. Now we set $\mathbf{d}^{\prime}=\mathcal{H}(\mathbf{c})$, so that $\mathbf{d}^{\prime}$ is invariant under $\mathcal{B}^{\prime}$ and $d_{2 m}=d_{2 m}^{\prime}$ for $m \geq 0$. Recall that $\mathcal{B}^{\prime}=D^{-1} \mathcal{B} D$, and that $D$ and $\mathcal{B}$ are linear. So $\mathcal{B}^{\prime}$ is also linear which implies that $\mathbf{d}^{\prime \prime}=\mathbf{d}^{\prime}-\mathbf{d}$ is also invariant under $\mathcal{B}^{\prime}$. Explicitly

$$
d_{n}^{\prime \prime}=\sum_{k=2}^{n}\left(\begin{array}{l}
n-1 \\
k-1
\end{array}\right) d_{k}^{\prime \prime}(-1)^{k}, n \geq 2
$$

where $d_{0}^{\prime \prime}=d_{1}^{\prime \prime}=d_{2 m}^{\prime \prime}=0$ for $m \geq 1$. Inductively it follows that $d_{n}^{\prime \prime}=0$ also for all odd $n \geq 0$, and so

$$
\mathbf{d}=\mathbf{d}^{\prime}=\mathcal{H}(\mathbf{c}) .
$$

This completes the proof of surjectivity.

\section{Examples and applications}

The well-known examples of invariant sequences under $\mathcal{B}$ have been given in Section 1 . A convenient reference for other examples is [4]. Here we will focus on two specific type of self-inverse sequences using results of Section 2.

First we need to recall a difference operator required in this section. We define the (forward) difference operator $\Delta$ as

$$
(\Delta \mathbf{d})(n)=d_{n+1}-d_{n}, \quad n \geq 0
$$

for any sequence $\mathbf{d}=\left\{d_{n}\right\}_{n \geq 0}$. If $f$ is a function on $\mathbb{N}$, we may write $(\Delta f)$ for $(\Delta \mathbf{d})$ where $\mathbf{d}=\{f(n)\}_{n \geq 0}$, i.e. $(\Delta f)(n)=f(n+1)-f(n)$ for $n \geq 0$.

We say that a sequence $\mathbf{d} \in \mathcal{A}_{0}$ is of polynomial type if there exists a polynomial $f(x)$ and $N \in \mathbb{N}$ such that $d_{n}=f(n)$ for all $n \geq N$. In this case we say that $\mathbf{d}$ is associated to $f(x)$. The existence of invariant sequences of polynomial type is already known. Indeed, if $f(x)$ is a polynomial of degree $r$ then the sequence $\left\{d_{n}\right\}_{n \geq 0}$ defined as

$$
d_{n}=(-1)^{n}\left(\Delta^{n} f\right)(0)+f(n), n \geq 0
$$

is an invariant sequence of polynomial type, [6, Theorem 3.2]. Note that in this case $d_{n}=f(n)$ for $n>r$. In particular, for any $r \geq 1$ the sequence

$$
d_{n}=(-1)^{n} n ! S(r, n)+n^{r}, n \geq 0
$$

is in $\mathcal{A}_{0}^{+}$for which $d_{n}=n^{r}$ if $n>r$ where $S(r, n)$ denotes the Stirling numbers of the second kind, [6, Example 3.3]. We say that a sequence $\mathbf{d}=\left\{d_{0}, d_{1}, \ldots\right\}$ is finitely supported if there exists $n \in \mathbb{N}$ such that $d_{n}=0$ for $n \geq N$.

Lemma 3.1. The only finitely supported sequence in $\mathcal{A}^{+}$is the zero sequence.

Proof. For an arbitrary sequence $\mathbf{d}$, if $\mathbf{d} \sim f(T)$ then

$$
\mathcal{B}(\mathbf{d}) \sim \frac{1}{1-T} f\left(\frac{-T}{1-T}\right) .
$$


If $\mathbf{d}$ is finitely supported then $f(T)$ is a polynomial. Additionally if $\mathbf{d} \in \mathcal{A}^{+}$then $f(T)=$ $\frac{1}{1-T} f\left(\frac{-T}{1-T}\right)$. But by considering the degree of $f(T)$ we see that this is possible if and only if $f(T)=0$.

Let $\mathbf{d}$ and $\mathbf{d}^{\prime}$ be of polynomial type, with $d_{n}=d_{n}^{\prime}=f(n)$ for $n \geq N$ for some polynomial $f$ of degree $r$ satisfying $f(0)=0$. Then $\left\{d_{n}-d_{n}^{\prime}\right\}_{n \geq 0}$ is also of polynomial type. But it is also a finitely supported sequence in $\mathcal{A}_{0}^{+}$, so it must be the zero sequence by Lemma 3.1. Hence there is a unique self-inverse sequence associated to any polynomial. Since $\left\{x^{r}\right\}_{r \geq 1}$ is a basis for the space of polynomials with zero constant term we have the following result.

Corollary 3.2. The sequences in (3.1) for $r \geq 1$ form a basis for the subspace of selfinverse sequences of polynomial type in $\mathcal{A}_{0}^{+}$.

Now we give another basis using the transform $\mathcal{H}$.

Theorem 3.3. Let $l \in \mathbb{N}$ with $l \geq 1$ and $f_{l}$ be the function on $\mathbb{N}$ defined as

$$
f_{l}(n)= \begin{cases}0, & n<2 l \\
\left(\begin{array}{c}
n-l-1 \\
l-1
\end{array}\right), & n \geq 2 l\end{cases}
$$

Then $\left\{n f_{l}(n)\right\}_{n \geq 0} \in \mathcal{A}_{0}^{+}$. Conversely, let $\mathbf{d}=\left\{d_{n}\right\}_{n \geq 0} \in \mathcal{A}_{0}^{+}$be of polynomial type. Suppose that $d_{n}=f(n)$ for $n \geq N$ where $f$ is a polynomial of degree $r$ satisfying $f(0)=0$. Then there exist unique $\alpha_{1}, \alpha_{2}, \ldots, \alpha_{r}$ such that

$$
\mathbf{d}=\left\{n \sum_{l=1}^{r} \alpha_{l} f_{l}(n)\right\}_{n \geq 0}
$$

Proof. Note that for a fixed $l \geq 1,\left\{n f_{l}(n)\right\}_{n \geq 0}$ is of polynomial type. Let $\mathbf{c}^{(l)} \in \mathcal{A}_{0}$ be the sequence for which

$$
\mathbf{c}_{k}^{(l)}= \begin{cases}0, & k \neq l \\ (-1)^{l}, & k=l\end{cases}
$$

Then we have $\left\{f_{l}(n)\right\}_{n \geq 0}=\mathcal{H}\left(\mathbf{c}^{(l)}\right)$. So the first part follows by Theorem 2.3.

To prove the converse, take any $\mathbf{d}$ and $f$ satisfying the hypothesis of the theorem. Without loss of generality we may assume that $N \geq 2 r$. For $l \geq 1$, the degree of the polynomial $x\left(\begin{array}{c}x-l-1 \\ l-1\end{array}\right)$ is $l$, so in particular, the set

$$
\left\{x\left(\begin{array}{c}
x-l-1 \\
l-1
\end{array}\right)\right\}_{l \geq 1}
$$

forms a basis for the space of polynomials with no constant term. Since $f(0)=0$ there exist unique $\alpha_{1}, \alpha_{2}, \ldots, \alpha_{r}$ such that

$$
f(x)=x \sum_{l=1}^{r} \alpha_{l}\left(\begin{array}{c}
x-l-1 \\
l-1
\end{array}\right) .
$$

Now for $n \geq N$, we have $d_{n}=f(n)=\sum_{l=1}^{r} \alpha_{l} n f_{l}(n)$. Also both of the sequences

$$
\left\{d_{n}\right\}_{\geq 0} \text { and }\left\{n \sum_{l=1}^{r} \alpha_{l} f_{l}(n)\right\}_{n \geq 0}
$$

are invariant under $\mathcal{B}$. So the difference

$$
\left\{d_{n}-n \sum_{l=1}^{r} \alpha_{l} f_{l}(n)\right\}_{n \geq 0}
$$

is a finitely supported sequence in $\mathcal{A}_{0}^{+}$which is necessarily the zero sequence by Lemma 3.1. 
So the set

$$
\left\{\left\{n f_{l}(n)\right\}_{n \geq 0} \mid l \geq 1\right\}
$$

is also a basis for the self-inverse sequences of polynomial type in $\mathcal{A}_{0}$. We also see that $\mathcal{H}$ maps the finitely supported sequence in $\mathcal{A}_{0}$ to self-inverse sequences of polynomial type in $\mathcal{A}_{0}$ bijectively.

Example 3.4. $l=1$ produces the sequence $d_{0}=d_{1}=0, d_{n}=n$ for $n \geq 2$. This elementary case is already known, [4]. Similarly the invariant sequence corresponding to $l=2$ is $d_{0}=d_{1}=d_{2}=d_{3}=0$ and $d_{n}=n(n-3)$ for $n \geq 4$.

Now we consider another type of self-inverse sequences which generalizes the Fibonacci sequence. First we need to introduce an operator $V$ on $\mathcal{A}_{0}$. For any $\mathbf{c} \in \mathcal{A}_{0}$ we define $V(\mathbf{c})$ as

$$
V(\mathbf{c})_{0}=0, V(\mathbf{c})_{n}=-c_{n+1} \text { for } n \geq 1
$$

i.e. if $\mathbf{c}=\left(0, c_{1}, c_{2}, \ldots\right)$ then $V(c)=\left(0,-c_{2},-c_{3}, \ldots\right)$. So the $L$-th power of $V$ is

$$
V^{L}(\mathbf{c})=\left(0,(-1)^{L} c_{1+L},(-1)^{L} c_{2+L}, \ldots\right)
$$

The images of the sequences $\mathbf{c}$ and $V(\mathbf{c})$ under $\mathcal{H}$ are related to each other.

Proposition 3.5. For any $\mathbf{c} \in \mathcal{A}_{0}$ and $n \geq 1$ we have that

$$
\mathcal{H}\left(V^{L}(\mathbf{c})\right)_{n}=\left(\Delta^{L} \mathcal{H}(\mathbf{c})\right)(L+n)
$$

Proof. We use induction on $L$. We set $\mathbf{d}=\mathcal{H}(\mathbf{c})$ and $\mathbf{d}^{(L)}=\mathcal{H}\left(V^{L}(\mathbf{c})\right)$ to simplify the notation. For $L=1$ the problem reduces to showing that $d_{n}^{(1)}=d_{n+2}-d_{n+1}$ for $n \geq 1$. This holds for $n=1$ as $\mathbf{d}$ is self-inverse and $d_{0}=d_{1}=0$.

For even $n$ we set $n=2 m$ for $m \geq 2$. Then

$$
\begin{aligned}
d_{2 m}-d_{2 m-1} & =\sum_{k=1}^{\lfloor 2 m / 2\rfloor}\left(\begin{array}{c}
2 m-k-1 \\
k-1
\end{array}\right)(-1)^{k} c_{k}-\sum_{k=1}^{\lfloor(2 m-1) / 2\rfloor}\left(\begin{array}{c}
2 m-k-2 \\
k-1
\end{array}\right)(-1)^{k} c_{k} \\
& =(-1)^{m} c_{m}+\sum_{k=2}^{m-1}\left(\begin{array}{c}
2 m-k-2 \\
k-2
\end{array}\right)(-1)^{k} c_{k} \\
& =(-1)^{m-1}\left(-c_{m}\right)+\sum_{k=1}^{m-2}\left(\begin{array}{c}
2 m-k-3 \\
k-1
\end{array}\right)(-1)^{k}\left(-c_{k+1}\right) \\
& =\sum_{k=1}^{\lfloor(2(m-1) / 2\rfloor}\left(\begin{array}{c}
2(m-1)-k-1 \\
k-1
\end{array}\right)(-1)^{k}\left(-c_{k+1}\right)=d_{2 m-2}^{(1)}
\end{aligned}
$$

In a similar way it also follows that $d_{2 m+1}-d_{2 m}=d_{2 m-1}^{(1)}$ for $m \geq 2$ which completes the proof of the claim for $L=1$.

Now assume that $d_{n}^{(L-1)}=\left(\Delta^{L-1} \mathbf{d}\right)(L-1+n)$. Since $\mathbf{d}^{(L)}=\mathcal{H}\left(V^{L}(\mathbf{c})\right)=\mathcal{H}\left(V\left(V^{L-1}(\mathbf{c})\right)\right)$ by using the case $L=1$ and the induction hypothesis we obtain that

$$
\begin{aligned}
d_{n}^{(L)} & =\mathcal{H}\left(V^{L-1}(\mathbf{c})\right)_{n+2}-\mathcal{H}\left(V^{L-1}(\mathbf{c})\right)_{n+1}=d_{n+2}^{(L-1)}-d_{n+1}^{(L-1)} \\
& =\left(\Delta^{L-1} \mathbf{d}\right)(L+1+n)-\left(\Delta^{L-1} \mathbf{d}\right)(L+n)=\left(\Delta^{L} \mathbf{d}\right)(L+n) .
\end{aligned}
$$

Corollary 3.6. Let $\mathbf{d} \in \mathcal{A}_{0}$ be invariant under $\mathcal{B}^{\prime}$. Then the sequence $\mathbf{d}^{\prime}=\left(d_{n}\right)_{n \geq 0}$ defined as

$$
d_{n}^{\prime}= \begin{cases}0, & n=0 \\ \left(\Delta^{L} \mathbf{d}\right)(L+n), & n>0\end{cases}
$$

is also invariant under $\mathcal{B}^{\prime}$. Equivalently, if $\left\{n d_{n}\right\}_{n \geq 0}$ is a self-inverse sequence in $\mathcal{A}_{0}$ then so is $\left\{n\left(\Delta^{L} \mathbf{d}\right)(L+n)\right\}_{n \geq 0}$ 
Proof. By Theorem 2.3, there exists $\mathbf{c} \in \mathcal{A}_{0}$ such that $\mathbf{d}=\mathcal{H}(\mathbf{c})$. Then the result follows by Proposition 3.5.

We may compare this result with [6]; if we set $a_{n}=n d_{n}$ in the notation of the above corollary, it follows by Theorem 3.4 of [6] that $\left\{\left(\Delta^{L} \mathbf{a}\right)(L+n)\right\}_{n \geq 0}$ is also self-inverse. But $\left\{\left(\Delta^{L} \mathbf{a}\right)(L+n)\right\}_{n \geq 0}$ and $\left\{n\left(\Delta^{L} \mathbf{d}\right)(L+n)\right\}_{n \geq 0}$ are obviously distinct sequences.

A related fact is that a sequence $\left\{n d_{n-1}\right\}$ for which

$$
d_{0}=0, d_{1}=1 \text { and }\left(\Delta^{1} \mathbf{d}\right)(1+n)=t d_{n}, n \geq 1
$$

where $t$ is an indeterminate is self-inverse, [4, Example 5 of Section 2]. In other words by $(2.1)$ the sequence $\left(0,0, d_{1}, d_{2}, \ldots\right)$ is invariant under $\mathcal{B}^{\prime}$. It is worthwhile to extend this fact to higher order difference equations $\left(\Delta^{L} \mathbf{d}\right)(L+n)=d_{n}$ for $L \geq 2$. Consider a sequence $\mathbf{d} \in \mathcal{A}_{0}$ which satisfies the difference equation

$$
\left(\Delta^{L} \mathbf{d}\right)(L+n)=d_{n}, n \geq 1 .
$$

Then $\mathbf{d}$ is uniquely determined by the initial conditions $d_{1}, d_{2}, \ldots, d_{2 L}$. It turns out that if the initial conditions on $d_{0}, d_{1}, d_{2}, \ldots, d_{2 L}$ obey the invariance under $\mathcal{B}^{\prime}$ then $\mathbf{d}$ is itself invariant under $\mathcal{B}^{\prime}$.

Theorem 3.7. Let $\mathbf{d} \in \mathcal{A}_{0}, L \in \mathbb{Z}$ with $L \geq 1$ and $t$ be an indeterminate. Suppose that

$$
\left(\Delta^{L} \mathbf{d}\right)(L+n)=t d_{n}, n \geq 1 .
$$

If $\mathcal{B}^{\prime}(d)_{n}=d_{n}$ for $0 \leq n \leq 2 L$ then $\mathcal{B}^{\prime}(\mathbf{d})=\mathbf{d}$.

Proof. Note that by assumption $d_{0}=d_{1}=0$. By using all even integers $n$ between 1 and $2 L$ we can recursively find $c_{1}, c_{2}, \ldots, c_{L}$ such that

$$
d_{n}=\sum_{k=1}^{\lfloor n / 2\rfloor}\left(\begin{array}{c}
n-k-1 \\
k-1
\end{array}\right)(-1)^{k} c_{k}, 1 \leq n \leq 2 L, n: \text { even }
$$

By hypothesis $\mathcal{B}^{\prime}(\mathbf{d})_{n}=d_{n}$ for all $n$ between 0 and $2 L$. So it follows that for any extension of $\left(0, c_{1}, c_{2}, \ldots c_{L}\right)$ to an infinite sequence $\mathbf{c}=\left(0, c_{1}, c_{2}, \ldots c_{L}, \ldots\right)$ we have that $\mathcal{H}(\mathbf{c})_{n}=d_{n}$ for all odd $n \in[0,2 L]$. In particular, we can choose $\mathbf{c}$ so that $V^{L}(\mathbf{c})=t \mathbf{c}$. Hence by Proposition 3.5 and linearity we see that

$$
t \mathcal{H}(\mathbf{c})_{n}=\mathcal{H}(t \mathbf{c})_{n}=\mathcal{H}\left(V^{L}(\mathbf{c})\right)_{n}=\left(\Delta^{L} \mathcal{H}(\mathbf{c})\right)(L+n), 1 \leq n \leq 2 L
$$

Now let $\mathbf{d}^{\prime}=\mathcal{H}(\mathbf{c})-\mathbf{d}$. Since the mapping $\mathbf{a} \mapsto\left\{\left(\Delta^{L} \mathbf{a}\right)(L+n)\right\}$ is linear, we see that $\mathbf{d}^{\prime}$ satisfies the difference equation

$$
\left(\Delta^{L} \mathbf{d}^{\prime}\right)(L+n)=t d_{n}^{\prime}, n \geq 1
$$

with the initial conditions $d_{0}^{\prime}=d_{1}^{\prime}=\ldots=d_{2 L}^{\prime}=0$. So $\mathbf{d}^{\prime}$ must be the zero sequence.

\section{References}

[1] M. Berstein and N.J.A. Sloane, Some canonical sequences of integers, Linear Algebra Appl. 226-228, 57-72, 1995.

[2] K. Boyadzhiev, Binomial Transform and The Backward Difference, Adv. Appl. Discrete Math. 13 (1), 43-63, 2014.

[3] H. Prodinger, Some Information about the Binomial Transform, Fibonacci Quart. 32, 412-415, 1994.

[4] Z. Sun, Invariant sequences under binomial transformation, Fibonacci Quart. 39 (4), 324-333, 2001.

[5] R. Taurosa and S. Mattarei, Congruences of Multiple Sums Involving Sequences Invariant Under the Binomial Transform, J. Integer Seq. 13 (5), Article 10.5.1, 2010.

[6] Y. Wang, Self-inverse sequences related to a binomial inverse pair, Fibonacci Quart. 43 (1), 46-52, 2005. 